\title{
Current Trends in Sustainability of Bitcoins and Related Blockchain Technology
}

\author{
Pasquale Giungato ${ }^{1, *}$ (1), Roberto Rana ${ }^{2}$, Angela Tarabella ${ }^{3}$ and Caterina Tricase ${ }^{2}$ \\ 1 Department of Chemistry, University of Bari, Bari 70125, Italy \\ 2 Department of Economics, University of Foggia, Foggia 71121, Italy; roberto.rana@unifg.it (R.R.); \\ caterina.tricase@unifg.it (C.T.) \\ 3 Department of Economics and Management, University of Pisa, Pisa 56126, Italy; angela.tarabella@unipi.it \\ * Correspondence: pasquale.giungato@uniba.it; Tel.: +39-099-4725-234
}

Received: 26 October 2017; Accepted: 27 November 2017; Published: 30 November 2017

\begin{abstract}
Bitcoin is a digital currency based on a peer-to-peer payment system managed by an open source software and characterized by lower transaction costs, greater security and scalability than fiat money and no need of a central bank. Despite criticisms about illegal uses and social consequences, it is attracting the interest of the scientific community. The purpose of this work is to define and evaluate the current trends of the literature concerned with the sustainability of bitcoin, considering the environmental impacts, social issues and economic aspects. From the analysis it emerges that the transition of the whole monetary system in the new cryptocurrency will result in an unacceptable amount of energy consumed to mine new bitcoins and to maintain the entire virtual monetary system, and probably bitcoin will remain a niche currency. Blockchain, which is the base for a distributed and democratically-sustained public ledger of the transactions, could foster new and challenging opportunities. Sharing the framework of medical data, energy generation and distribution in micro-grids at the citizen level, block-stack and new state-driven cryptocurrencies, may benefit from the wide spread of blockchain-based transactions. Under the perspective of its being a driver of social change, bitcoins and related blockchain technologies may overcome the issues highlighted by numerous detractors.
\end{abstract}

Keywords: bitcoin; sustainability; monetary exchanges; digital currency; energy consumption

\section{Introduction}

Since the introduction of the first IBM personal computer in 1975, and later in 1993 with the diffusion of the "internet", technology entered homes and spread widely over the world as a new digital revolution that brought sophisticated and distant technologies within everyone's reach, such as photo development, music listening and authoring, and home banking. The introduction of bitcoin in 2009 seems to represent a new starting revolution in the way we make money exchanges and value creation [1]. Bitcoin can be considered as the next stage in the process of money evolution: an electronic money in no way connected to the objects of the material world, with several advantages when compared to other forms of money [2].

Bitcoin is a digital currency based on a payment system that relies on a peer-to-peer network created as an open source program in 2008 and launched in 2009 by a developer named Satoshi Nakamoto, but this may be a pseudonym behind which another individual or group may hide. The creation and transfer of this virtual currency is made under cryptographic connections for this reason Bitcoin is also referred to as "cryptocurrency". The mechanism of creation, distribution and maintenance is not managed by a central bank, but is decentralized in a worldwide community sharing a peer-to-peer connection and using a software that manage exchanges like a digital wallet. The transfer 
of currency from one wallet to another is simply an exchange of digital bits without involving paper, coins, credit or debit cards, bank accounts, security accounts and no collateral. In addition, bitcoins rely on dedicated rigs of personal computers, mining for new coins through a series of tasks that require considerable computational power.

Presently, although bitcoin is considered a currency that could overcome limits of the fiat money, there are some issues related to its sustainability. In this regard, bitcoins may be viewed from different angles: the economic, social and environmental points of view [3]. First, a precise definition of the sustainability of bitcoins is needed. We must consider that bitcoins are entities living in a different ecological ecosystem: the digital ecosystem. A digital ecosystem consists of all hardware devices, program files, and data files that the user needs and it shares with other users. The ecosystem as defined also includes the social elements which led to the creation and use of digital elements like bitcoins as no-material technological objects [4]. The digital resources we mentioned are more properly defined as "digital artifacts", "entities that consist of strings of 0 and 1, which can be interpreted by technical devices, like computers, to provide some meaning" [5]. As bitcoins have an economic value it can be considered a "digital good" in which the computing capacity defines both the scarcity of the good and the raising of its economic value [6]. Indeed, bitcoin's increasing value is due to its rocketing popularity in recent years. For instance, in 2009 there were fewer than 10,000 transactions in bitcoin, whereas by May of 2017 this number had exceeded 300,000. Consequently, the economic value of bitcoin in May 2017 had reached even more than 5000 United States dollars (USD) [7].

The economic standpoint relies on the ingenious mechanism of transaction in which there is not a third party which guarantees an exchange but the transaction happens directly between the exchangers and the system provides a cryptographic proof of the transaction. Moreover, bitcoin creation is not indefinite as the number of bitcoins is limited to a definite number, generating an inflation due to currency scarcity and making it a store of intrinsic value as gold and silver. At the present day about 25 new bitcoins are generated every ten minutes, and that number will progressively be halved every four years [8]. Bitcoin mining is increasingly difficult requiring both more people or PCs and complex algorithms: at the present only the most powerful computational networks, with several central processing units working together, are able to create additional coins. Secondly according to some authors, the bitcoin protocol as it was planned, consumes increasing amounts of energy and natural resources, as well as being used by illegal activities [9].

In this context, the present work is intended to focus on the current trends in exploring the sustainability concepts related to bitcoin diffusion. In particular, because the literature has focused basically on economic aspects, the present review will consider the environmental and social sustainability of this cryptocurrency and its future trends.

\section{Materials and Methods}

The literature review was conducted by using as the keywords for online research the words "bitcoin", "digital currency", "cryptocurrency" and "virtual currency", with "sustainability" in the databases Scopus, ISI Web of Science and Google Scholar (Table 1).

Table 1. Number of papers that considers the terms "bitcoin", "digital currency", "cryptocurrency" and "virtual currency" plus sustainability in the Scopus, Web of Science and Google Scholar databases.

\begin{tabular}{|c|c|c|c|c|c|c|c|c|}
\hline & Bitcoin & $\begin{array}{l}\text { Bitcoin + } \\
\text { Sustain }\end{array}$ & $\begin{array}{l}\text { "Digital } \\
\text { Currency" }\end{array}$ & $\begin{array}{c}\text { "Digital } \\
\text { Currency" + } \\
\text { Sustain. }\end{array}$ & Cryptocurrency & $\begin{array}{c}\text { Cryptocurrency + } \\
\text { Sustain. }\end{array}$ & $\begin{array}{l}\text { Virtual } \\
\text { Currency }\end{array}$ & $\begin{array}{c}\text { Virtual } \\
\text { Currency }+ \\
\text { Sustain. }\end{array}$ \\
\hline Scopus & 819 & 5 & 553 & 1 & 217 & 4 & 254 & 1 \\
\hline Web of Science & 502 & 4 & 377 & 0 & 124 & 2 & 147 & 1 \\
\hline Google scholar & 24,800 & 1780 & 6480 & 1150 & 6490 & 563 & 14,500 & 2110 \\
\hline
\end{tabular}

Table 1 show that generally a small number of papers have been published considering the sustainability of bitcoin, digital currency, cryptocurrency or virtual currency. Furthermore, the literature 
research showed that in all databases consulted the majority of papers have been published in the last three years $(2015,2016$ and 2017), probably because the bitcoin technology is becoming more and more appreciated and globally used.

\section{Environmental Aspects of Sustainability}

The environmental sustainability of bitcoins is a controversial question as the system has been built in a way almost like the mining of a natural resource: costs and efforts rise as the system reaches the ultimate resource limit. In other words, just as the mining of copper or gold or crude oil becomes more and more expensive and time consuming as we approach the finite quantitative limit, so also the "mining" of new bitcoin requires more and more hardware resources necessary to "mine" each bitcoin when approaching the capped limit of the bitcoin system. Bitcoin is based on the "blockchain technology" which relies on highly secure cryptographic algorithms and sophisticated peer-to-peer technologies, which poses a challenge to both the usability and diffusion of bitcoins. Bitcoin mining consists in the addition of a pool of valid transactions in the blockchain validated by each node. Once a node has collected a number of valid transactions in a block, the node computes a cryptographic hash of that block to comply with a definite constraint. To find a hash the meets the constraint is a computing-intensive task. The system is elastic as the number of attempts required to find a valid hash varies depending upon the computing capacity of entire network, but it is regulated to require an average of ten minutes to generate a new valid block and reward the miners.

The rate of emission of bitcoins (called "bitcoin mining") has an inflexible algorithmic limitation starting from zero (really 50) and increasing rapidly but the increasing rate slow down constantly: after the issue of 10.5 million bitcoins, its emission rate will decrease by half, after 15,750,000 bitcoins, the emission rate will halve and so on, reaching a limited capped value of the total bitcoin amount of 21 million. For this reasons bitcoin mining does not follow a logistic distribution, like the statistical Hubbert's peak distribution functions, but follows a logarithmic growth. Nowadays there are about slight over 16 million bitcoins mined, Figure 1 [7].

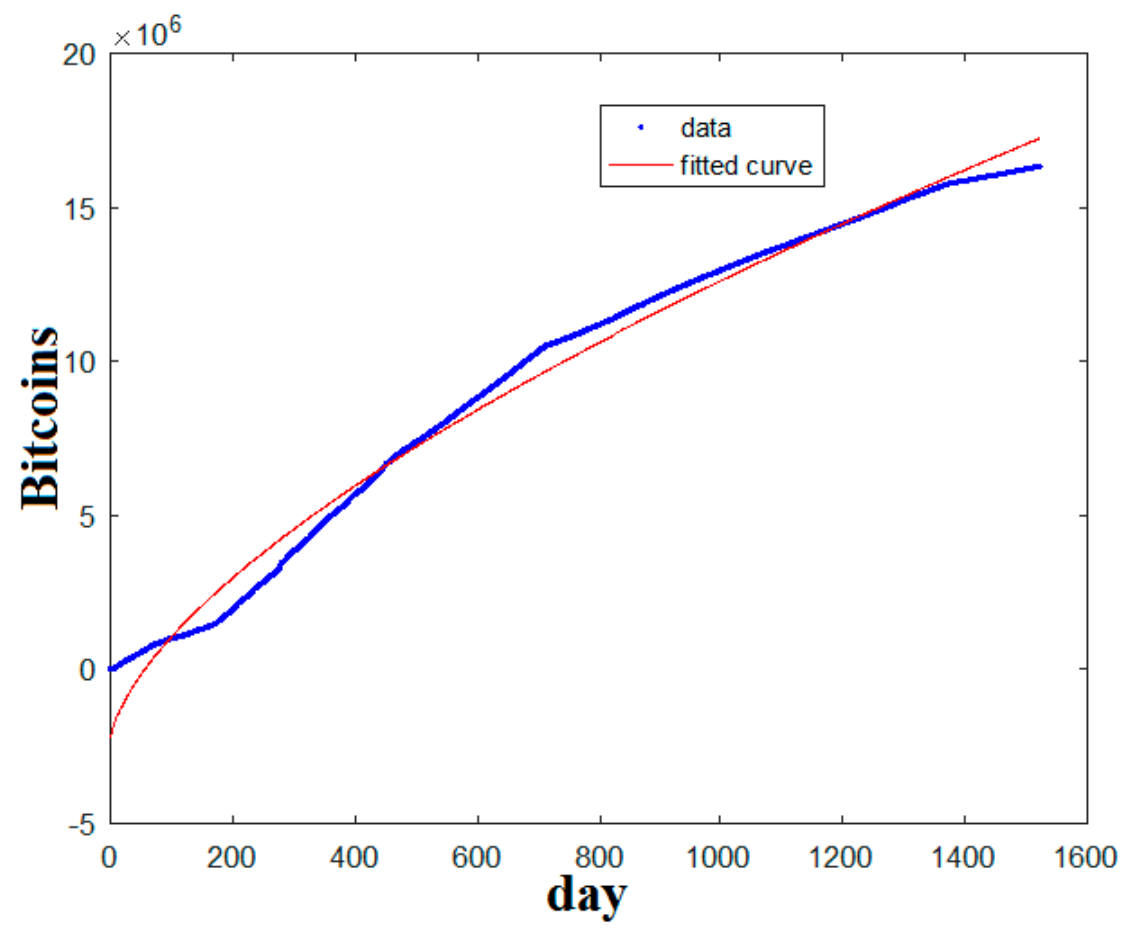

Figure 1. Bitcoins mined per day (1523 data every two days from 3 January 2009 to 5 May 2017) [7]. Source: power law curve fitting with Matlab R2016a. 
Every block introduces 50 new coins in the system, a number halving every 210,000 blocks, defining a geometric series which converges to a capped limit of about 21 million bitcoins (a number subjected to a slight lowering due to rounding but embedded in the algorithm protocol). Such a geometric series may be modelized as a power function as forecast by the algorithm or may follow a logistic distribution as in the case of crude oil or other energetic commodities. The results of fitting the total number of mined bitcoins versus time with both a power and logistic curve are reported in Figure 1 and Table 2.

Table 2. Curve fitting and goodness-of-fit statistics [7].

\begin{tabular}{ccc}
\hline Distribution & Power Law & Logistic \\
\hline Formula & $\mathrm{f}(\mathrm{x})=\mathrm{a} \mathrm{x}^{\mathrm{b}}+\mathrm{c}$ & $\mathrm{f}(\mathrm{x})=$ const $/\left[1+\exp \left(-\mathrm{X}{ }^{*} \mathrm{~b}\right)\right]$ \\
SSE & $4.719 \times 10^{+14}$ & $2.45 \times 10^{+15}$ \\
$\mathrm{R}^{2}$ & 0.9881 & 0.8396 \\
Adjusted-R & 0.9881 & 0.8395 \\
RMSE & $5.572 \times 10^{+5}$ & $1.269 \times 10^{+6}$
\end{tabular}

Legend: SSE $=$ Sum of squares due to error, RMSE $=$ Root mean squared error. $X=$ day, $f(x)=$ Bitcoins. Source: Matlab R2016a, and Gretl-http:/ / gretl.sourceforge.net/.

The number of bitcoins mined from the network has increased following more a power law than a logistic regression, as evidenced by the lower SSE (sum of squares due to error) and RMSE (root mean squared error) values in the first case. These results state clearly that the increasing demand of electrical current and computing power do not represent a limiting factor in the bitcoin mining capacity of the network. The generation of new bitcoins follows the generation algorithm imposing a reduction of mining rate every four years. From this point of view, bitcoin mining is not like an energetic commodity mining, although it requires energy and material to maintain increasing computing power.

Currently the limited number of bitcoins that can be created does not pose a serious threat to the diffusion of this cryptocurrency, as bitcoins can be divided into to eight decimal places, or 100 million "Satoshi's" like a dollar is divisible in cents; eventually the problem of divisibility will arise in the next 30-50 years if bitcoin replaces all the existing currencies [10].

Mc Cook [11] stated that, at present, the environmental costs of bitcoin mining are less than paper money, gold and banking systems (Table 3).

Table 3. Environmental costs of bitcoin mining compared with other systems.

\begin{tabular}{cc}
\hline Currency & PJ/y \\
\hline Bitcoin & 3.97 \\
Paper currency and minting & 39.6 \\
Gold mining & 475 \\
Gold recycling & 25 \\
Banking system & 2340 \\
\hline
\end{tabular}

The study considered only energy consumption, in comparison with other systems, excluding producing gold mining machinery, storing and transporting, constructing about 600,000 bank branches in the worlds employing about 7 million persons, and including only operational energy used. Undoubtedly bitcoin mining consumes less energy than the other systems like gold mining, banknotes, coins and the banking system, but considering the transition of all monetary systems into the new cryptocurrency, this will result in a huge amount of energy consumed to mine new bitcoins and to maintain the entire virtual monetary system at the actual bitcoin mining rate. Alternatives to bitcoin and fiat currencies are represented by polymer-based banknotes. A "cradle-to-grave" life cycle assessment (LCA) of the current cotton paper bank notes in Canada compared with a new polymer bank note prototype (choosing the provision of 2000 Canadian Dollar banknotes of cash value over a 
time span of 7.5 years) has shown the benefits of polymer banknotes over cotton for all main phases of the life cycle: manufacturing (since they have to be produced 2.5 fewer times than the cotton paper bank note), distribution (they have to be distributed 2.5 less times and their weight is lighter) and for end-of-life (since the carbon contained in cotton paper bank notes is released as GHG (Green-House Gas) in landfill) [12]. However, no assessment has been done at present of bitcoin, so is not clear if it is sustainable or not. For instance, Loviscach [13] has proposed two fundamental aspects to be taken into account in assessing the environmental impact of bitcoin, such as (1) computer energy consumption (expressed in $\mathrm{kWh}$ ) for computing, networking and cooling (2) disposal of e-waste produced. The "Long Future Foundation", an Australian sustainability organism, recently expressed worries about the energy consumption used and greenhouse gases (GHGs) released by bitcoin mining. The foundation suggests that $40 \%$ of the bitcoin is spent on electricity costs to mine it. Furthermore, the cryptocurrency could have generated 4 gigatons of GHGs, almost $13 \%$ of global releases per annum (4.6 $\mathrm{CO}_{2 \mathrm{eq}}$ emitted for each dollar spent on bitcoin's electricity). The foundation also recommend that in order to assess the carbon footprint of bitcoin, three factors should be considered: (1) the electricity consumption of the network (electricity consumed per gigahash, multiplied by the total number of gigahashes produced); (2) the carbon intensity of the consumed electricity (this depends on the energy mix of a nation and decreases if renewable energy sources are used and increases fossil fuels are used; (3) the price of bitcoin. The increase in the economic value of bitcoin can drive people to extend mining activities and consequently to increase energy consumption [14].

O'Dwyer, and Malone [15] have estimated the energy consumption, finding that the entire Bitcoin mining network is on par with Ireland for electricity consumption. According to Dimitriou and Karame [16], this is not entirely true, since the increased economic and technological costs to obtain virtual coin requires the creation of increasing efficiency for the bitcoin network. Therefore, the statement that the cryptocurrency "trade and consume a huge quantity of energy" seems not to be true. Thus, if a user generates blocks more efficiently and speedily than others, they can reduce the energy consumption and receive a great reward (such as new bitcoin and/or transaction fees), pushing the system towards an increasingly low energy consumption [15]. In other words, the inefficient miners become unprofitable and are replaced by more efficient ones. To ascertain whether the bitcoin infrastructure could reach a future sustainable rate of demand for energy and materials should be assessed, considering that cryptocurrencies will replace the actual monetary system. As the computing capacity needed to mine new bitcoins increases steadily, it is obvious to consider not only the energy but also the machinery involved. Every attempt to investigate materials and energy consumption based on the computing capacity of the system will bring about pessimistic results regarding the environmental sustainability of every cryptocurrency like bitcoin $[17,18]$.

As Figure 1 confirms, the cumulated bitcoin mining does not follow a statistical distribution like that of crude oil or other energetic commodities (which is a logistic distribution), but a power law, in which the rate of approaching the carrying capacity of the system is higher than in the case of crude oil or other non-renewable commodities. This leads to the belief that at the actual increasing rate of bitcoin mining, the system will too rapidly reach its carrying capacity. In this case, the sustainability of bitcoin could be assured only by a lower growth rate than that forecast by the author of the algorithm, resembling that of crude oil or coal. This lowering rate could arise from the requested hardware and software infrastructure needed to mine new bitcoins, as the cumulated mining production approaches the capped value of 21 million. The reasons could be the same as in the case of energetic not-renewable commodities, as the expenses in terms of infrastructure and energy used to explore a new deposit of crude oil or coal will increase as the cumulated production of the commodity approaches the ultimate reserves. In this case, there are fewer bitcoins to be mined, transaction fees will increase and become the sole source of income for miners, needed even after reaching the capped limit, because new blocks will always be needed to verify bitcoin transactions and keep the infrastructure running.

The European Union is evaluating the advantages and disadvantages deriving from the new tools related to the collaborative internet, such as big/open data, cryptocurrency and additive manufacturing. 
These aspects are important since their impacts are not restricted to where economic activities take place, but they are connected also to where the people live or work, namely everywhere [19].

\section{Social Aspects of Sustainability}

Bitcoin's transactions rely on a distributed database of records that can be considered a public peer-to-peer ledger of all the transactions shared among all the participants. The security of the system is assured by a digital distributed consensus of most of the participants in the system and the information can never be erased. The underlying blockchain technology developed a sort of democratic open and scalable digital economy in which all the transaction between two or more economic entities become traceable. Considering the number of transactions (as contracts or documents) among business and marketing entities and the expenses that companies experience in maintaining paper-based archives, this technology of distributed and democratically-sustained public ledgers of the transactions opens new and challenging opportunities for the economic system. For these reasons, interest in this technology is rising from the banking and financial system, and its social implications for changing organizations are enormous [20,21]. Blockchain technology is attracting massive attention and triggering multiple projects in different industries. Blockchain-based sharing frameworks of medical data have been proposed, which can conveniently and efficiently track records of access to sensitive medical data stored in the cloud using the immutability and built-in autonomy properties of the blockchain. This blockchain-based access control system can facilitate the exchange of information between healthcare and research institutions as it sufficiently controls the access to medical data stored and processed on cloud systems and it offers secure cryptographic techniques to identify and authenticate users that have access to medical data, keeping track of all activities [22]. From this point of view, the social sustainability of the technology underlying bitcoin is assured from the innovations that it could generate in the economic system. For instance, it could have relevant impacts on targeted agriculture value chains in developing countries, where farmers live and work in off-grid rural communities. In November 2016, the United Nations (UN) started to discuss how bitcoin technology can contribute to implementing sustainable development. It considers this technology as an important tool to improve the applications of microfinance or the remittances for the livelihoods of rural smallholder farmers and their families, and consequently to contribute to reaching the sustainable development goals [23,24]. Bitcoin technology could reduce less than $3 \%$ of the transaction costs for migrant remittances and eliminate remittance corridors with costs higher than $5 \%$ by 2030 [25]. However, the spread of this technology is difficult to ensure in developing countries characterized by a population with relatively low levels of education, literacy and poor infrastructure to promote in these territories the long-term sustainability that is necessary to improve these aspects [26].

Another interesting case of the application of the blockchain technology is in energy generation and distribution. The diffusion of micro-wind, solar, and micro-hydro small power generating systems is rapidly disrupting the traditional centralized one-directional power grid syste towards micro-grids at the citizen level. With the mass installation of micro-wind, solar and hydro generating systems coupled with battery storage and sometimes integrated with traditional fossil fuel generators, citizens are moving from consumers to consumer-producer entities aiming to reduce their power bills and to sell their excess power to other local users via micro utilities, enabling technologies such as the blockchain [27]. Social acceptance of this transition process starting from the widespread adoption of small-scale technologies like rooftop installations of solar photovoltaic or micro-wind generators and the downstream impact of the actual centralized electricity grids remains unclear. The citizen's utilities, as they are called, these new entities in which consumer-producers are growing, needs a technology that ensure communications and data exchange without a centralized server but relying on a distributed public ledger of all energy transactions. Companies such as Grid Singularity, Solar Coin and Ethereum are using the blockchain technology to transact electricity among citizen's utilities and microgrids, which can benefit from using a commonly-used database for managing energetic 
and financial transactions in a distributed, consensus-driven infrastructure, enabling trust between counterparties [28,29].

Bitcoin and other forms of cryptocurrency are particularly attractive for libertarian and/or anarchist sympathizers, who want to see money removed from the control of governments or a central bank. On 13 August 2014, the Declaration of Bitcoin Independence was released, focusing mainly on the new currency's political significance and on its anonymity [30,31]. As a matter of fact, bitcoin is a decentralized network of individuals recording every transaction on a virtual ledger called the blockchain. When a virtual money transaction (block) is "confirmed" (validated) a number code is added connecting the new block with the previous one. As it is impossible to modify the sequence of chains in the blockchain it is impossible to reuse a bitcoin that has been already spent. The bitcoin system today allows about three transactions per second, whereas VISA circuits about 60 thousand, thus creating some perplexities in the financial world about the widespread use of bitcoins [32].

Although independence represents one of the most important aspects of bitcoin, on the other hand it creates an intrinsic rigidity. If a procedure changes without user approval (e.g., from bitcoin core-BC, to bitcoin unlimited-BU), a different cryptocurrency is de facto created. In this condition, the logic of market competition between alternative solutions becomes the only possibility and the government of the original protocol is abandoned. Dodd (2017) states that real independence of the bitcoin system is not achievable, because its anarchism is compromised by the way that the system operates in practice: it favors the most powerful producers of the currency to become even more powerful. In other words, this means that it incentivizes monopolistic practices and makes bitcoin not a "distributed network" but a way to support strong tendencies towards the centralization of monetary production. Some facts show that Bitcoin could represent an unsustainable currency from a social point of view, since it can be use by hacking and fraudulent activities or by criminal associations to trade illegally, including drugs, weapons, etc. These aspects seem to be connected to its anonymity, although a positive perspective could arise when considering that bitcoin will prevent some governments from printing money at will and then using that to buy weapons [33]. The close association between anonymity and illegal activities is not true in principle. Indeed, all bitcoin transactions are stored publicly and permanently on the network. The bitcoin system presented in these terms is not a tool of anonymity, but rather a transparent and trackable means of information shared across a network that does not rely on a centralized agency [34]. However, this is true in principle because it can be used anonymously by applying a bitcoin address once. Therefore, to improve the legal image of bitcoin this problem should be improved and solved. The induction of bitcoin may be a very powerful tool to fight poverty. For example, in Cyprus people began to convert money from their bank accounts to bitcoin when the government announced a plan to sequester cash from the country's bank accounts.

\section{Economic Aspects of Sustainability}

Bitcoin mining started by using the central processing units of the hardware in use in 2009, but with the increase of computational needs the miners shifted towards more powerful graphic process units, which benefitted from higher parallelism in computing, at the end of 2010. Field programmable gate arrays (FPGAs) and, later in 2013, specifically designed processing units (application-specific integrated circuits, ASICs) containing dedicated hardware optimized to solve hashing retrieve from the algorithm were introduced. The hash rate has increased from $10^{5}$ hash $/ \mathrm{s}$ to $10^{8}$ hash $/ \mathrm{s}$ of the first-generation CPUs (Central Processing Units) to $10^{10}-10^{13}$ hash/s of the most performant ASICs unit. Consequently, the energy efficiency also sank from $10^{4}-10^{5}$ hash $/ \mathrm{J}$ to $10^{8}-10^{10} \mathrm{hash} / \mathrm{J}$. The revenue of miners due to the reward in searching for a valid block of transactions and a valid hash dropped constantly since the launch of the cryptocurrency. To maintain profitable mining revenue, the miners switched to more powerful and less energy-demanding hardware considering also the costs of electricity, which vary depending on the country of operation, and nowadays is below $10^{-3}$ USD per Gh/s [35]. Since its introduction to the global market, bitcoin's network has grown rapidly and at present numerous economic activities accept bitcoins (e.g., Amazon, by using gifts bought from 
third parties), music repositories or gambling websites, game or software providers, online businesses (e.g., Expedia, WordPress, Mega etc.). One of the major threat regarding the spread of bitcoins in the global payment system is the relatively slow rate of transactions, as the blockchain mechanism requires that a block should be validated to reward miners for their efforts, and the system is elastic to create a delay time to insert a new valid block in the blockchain; this is not competitive with credit cards and banking circuits. To match the cycles of growth and depression of economies and to maintain inflation to a physiological amount, the k-percent, Milton Friedman monetary theory considers essential the role of a central bank in increasing the money supply by a constant percentage every year, to prevent excessive inflation. Bitcoin mining supplies new cryptocurrencies in a logarithmic growth in which the increase rate will slow down, generating a deflationary bias to the value of goods and services. Smaller bitcoin supply may be overcome by an increase in the velocity of bitcoin transactions to maintain constant inflation [36].

\section{Future Trends in Blockchain Technology}

According to the recent development of bitcoin technology applications, the blockchain could represents one of the most important aspects that can be applied in many sectors in the future. This is because the content of the blockchain is information and its use is more versatile than the cryptocurrencies. Kell et al. [37] affirm that this innovation appears to represent a promising catalyst for achieving global sustainable development targets. For instance, blockchain technology may contribute to building a smart city through the development of shared economic services [38]. In fact, to implement trust-free sharing services in a smart city, it is necessary to consider security aspects (such as confidentiality, availability, and integrity) which represent one of the principal characteristic of blockchain-based technology. This is because a blockchain is a decentralized protocol where all information is confidential and the availability of data does not rely on any third parties. Moreover, integrity is ensured since this technology can be regarded as a distributed file system where participants keep copies of files and agree to changes by consensus.

Faber and Hadders [39] have underlined the potential of blockchain technology to create an infrastructure to implement "new social contracts for sustainability", contributing the promotion of the transition to sustainable development. They state that blockchains provide infrastructure for data transactions that does not require centralized functions supporting true connections and relationships among people to solve problems. Therefore, this technology overcomes the current business model, which is principally manage by old mainstream bureaucratic and power institutions like political parties, banks, local governments, etc.

Blockchain technology can also be applied in the decentralization of the DNS (domain name system) management called "block-stack", an alternate, DNS-like system that replace DNS root servers with a blockchain technology for mapping domain names to DNS records. DNS is like the phonebook of the internet, as when typing a web address a DNS server translates that address and returns an IP (internet protocol) address. DNS servers are controlled by governments and large corporations and this centralized control may foster abuses of power in censoring activities, hijacking, spying and hacker attacks. The blockchain technology may foster the decentralization, security, censorship resistance, privacy and speed of certain components of the internet's infrastructure, such as DNS servers. Given that blockchains do not have central points of trust, a blockchain-based DNS is much harder to censor [40]. Moreover, the blockchain technology may also be used to decentralize TLS (transport layer security) certificate validation, backed by blockchain consensus. TLS is a cryptographic protocol that provides communications security over a computer network. Several versions of the protocol have been released and it is widely used in web browsing, email, web-fax, instant messaging, and voice-over-IP (VoIP). In other word TLS will secure all communications between servers and web browsers on a personal computer. File signatures, voting, bonds/stocks/shares, web of trust, notary services, and proof of existence are interesting and futuristic applications of blockchains, and open source experimental applications are developing on the web. Interesting experiments are being 
developed in highly-digitalized countries, such as Estonia, where the launch of a digital citizenship (e-residency), a digital identity for non-Estonians that offers access to services like banking, payment processing and taxation and of a new government-banked cryptocurrency (Estcoin) with no possibility of counterfeiting and use for illegal purposes, will probably foster the transition towards a truly digital, borderless society [41].

\section{Conclusions}

Proposed by a developer hiding behind the pseudonym Satoshi Nakamoto in 2009, bitcoin is a digital currency based on a peer-to-peer payment system created as an open source software. The creation and transfer of this virtual currency is made under cryptographic connections, and for this reason bitcoin is also referred to as a "cryptocurrency". Bitcoin is based on "blockchain technology", which relies on highly secure cryptographic algorithms and sophisticated peer-to-peer technologies that are the base of a distributed and democratically-sustained public ledger of transactions. Although a definition of sustainability has no full applicability to cryptocurrencies, we should consider that bitcoins are entities living in a different ecological ecosystem, the digital one, which consists of all hardware devices, program and data files that a user needs to share with other users Bitcoins are non-material technological objects. Bitcoins as virtual money can be environmentally sustainable since it requires few natural resources (e.g., fossil fuels) to sustain and maintain the exchange system of value in comparison with other payment or banking circuits (like credit cards). The environmental costs of bitcoin's mining and network maintenance depends on the rate of diffusion into the monetary system, as considering only the energetic costs, the system seems to be less energy consuming than gold mining or the banking system. As a substitute for the present monetary system, bitcoin will remain a niche market as bitcoin mining will consume more and more energy, and considering the transition of all monetary systems to the new cryptocurrency, this would result in a huge amount of energy consumed to maintain the entire virtual monetary system at the actual bitcoin mining rate. To maintain profitable mining revenues, miners use more and more powerful and less energy-demanding hardware, starting from the CPU, and passing through GPU, FPGAs and recently ASICs to follow economic sustainability and placing bitcoin farmers in the countries with the cheapest electricity prices. Some skepticism regarding bitcoin's social sustainability has been addressed, since it can be use by hackers and for fraudulent activities or by criminal associations to trade drugs, weapons, etc. These aspects seem to be connected to the anonymity, which is a primary attraction for libertarian and/or anarchist sympathizers, and hackers and those wishing to carry out fraudulent activities. Blockchain technology seems to be an interesting application for a sharing framework for medical data, energy generation and distribution among micro-wind, solar, micro-hydro small power generating systems connected as micro-grids at the citizen level and for the management of legal transactions among companies. From the perspective of a large-scale social experiment, bitcoin and related blockchain technologies will be drivers of social changes and from this perspective, the sustainability of this digital currency will be revalued.

Acknowledgments: This paper is financially supported by Department of Economics and Management, University of Pisa, Italy.

Author Contributions: Giungato and Rana conceived and designed the review, and performed the searches; Tricase and Tarabella analyzed the papers; all contributed with analysis tools and wrote the paper.

Conflicts of Interest: The authors declare no conflict of interest.

\section{References}

1. Marc, A. Why Bitcoins matters. New York Times, 21 January 2014. Available online: http:/ / dealbook.nytimes. com/2014/01/21/why-Bitcoin-matters (accessed on 5 May 2017).

2. Vlasov, A.V. The evolution of e-money. Eur. Res. Stud. J. 2017, 20, 215-224. 
3. Dalal, N. Exploring the Bitcoin System: A Complex Econo-Sociotechnical Systems (CEST) Perspective. Int. J. Concept. Manag. Soc. Sci. 2014, 2, 47-51.

4. Faulkner, P.; Runde, J. Technological objects, social positions, and the transformational model of social activity. MIS Q. 2013, 37, 803-818. [CrossRef]

5. Stuermer, M.; Abu-Tayeh, G.; Myrach, T. Digital sustainability: Basic conditions for sustainable digital artifacts and their ecosystems. Sustain. Sci. 2017, 12, 247-262. [CrossRef]

6. Quah, D. Digital Goods and the New Economy. 2003. Available online: https:/ /ssrn.com/abstract=410604 (accessed on 3 May 2017).

7. Blockchain. Available online: https:/ /blockchain.info (accessed on 3 May 2017).

8. Dodd, N. Theory, Culture \& Society. In The Social Life of Bitcoin; Sage Publishing: Thousand Oaks, CA, USA, 2017; ISSN 0263-2764. in press; Available online: http: / / eprints.lse.ac.uk (accessed on 3 May 2017).

9. Li, X.; Wang, A.C. The technology and economic determinants of cryptocurrency exchange rates: The case of Bitcoin. Decis. Support Syst. 2017, 95, 49-60. [CrossRef]

10. Mc Cook, H. Busting Bitcoin Myths: Myth \#001-There Aren't Enough Bitcoins. 2014. Available online: https:/ / dealingwithdisruption.com/2014/04/16/myth001 (accessed on 3 May 2017).

11. Mc Cook, H. An Order-of-Magnitude Estimate of the Relative Sustainability of the Bitcoin Network, 3rd ed.; Working Paper; 11 February 2015. Available online: https://cdn.panteracapital.com/wp-content/uploads/ The-Relative-Sustainability-of-the-Bitcoin-Network.pdf (accessed on 3 May 2017).

12. Marincovic, C. Life Cycle Assessment of Canada's Polymer Bank Notes and Cotton-Paper Bank Notes-Final Report; PE Americas: Boston, MA, USA, 2011. Available online: http://www.bankofcanada.ca/wp-content/ uploads/2011/06/Life-Cycle-Assessment-of-Polymer-and-Cotton-Paper-Bank-Notes_opt.pdf (accessed on 3 May 2017).

13. Loviscach, J. The Environmental Cost of Bitcoin. 2012. Available online: http://webcache.googleusercontent. com/search?q=cache:InY5ueoiTFMJ:www.j317h.de/talks /2012-09-20_Environmental_Cost_of_Bitcoin. $\mathrm{pdf}+\& \mathrm{~cd}=2 \& \mathrm{hl}=\mathrm{it} \& \mathrm{ct}=\mathrm{clnk} \& \mathrm{gl}=\mathrm{it}$ (accessed on 3 May 2017).

14. Anonymous. Bitcoin and the Planet-Bitcarbo. Bitcoin's Carbon Footprint. 2017. Available online: http: / / longfuture.org/Bitcoin (accessed on 14 June 2017).

15. O’Dwyer, K.J.; Malone, D. Bitcoin Mining and its Energy Footprint. In Proceedings of the ISSC Irish Signals \& Systems Conference and China-Ireland International Conference on Information and Communications Technologies (ISSC 2014/CIICT 2014), Limerick, Ireland, 26-27 June 2014.

16. Dimitriou, T.; Karame, G. Privacy-friendly tasking and trading of energy in smart grids. In Proceedings of the 28th Annual ACM Symposium on Applied Computing, Coimbra, Portugal, 18-22 March 2013; ACM: New York, NY, USA, 2013; pp. 652-659.

17. Malmo, C. Bitcoin Is Unsustainable. Motherboard, 2015. Available online: https://motherboard.vice.com/ en_us/article/Bitcoin-is-unsustainable (accessed on 5 May 2017).

18. Deetman, S. Bitcoin Could Consume as Much Electricity as Denmark by 2020. Motherboard, 2016. Available online: https://motherboard.vice.com/en_us/article/Bitcoin-could-consume-as-muchelectricity-as-denmark-by-2020 (accessed on 3 May 2017).

19. Achilleopoulos, N.; Bengtsson, J.E.; Crehan, P.; Giuliano, A.; Soldatos, J. The Collaborative Economy-Impact and Potential of Collaborative Internet and Additive Manufacturing; Robertshaw, S., Ed.; European Parliament: Brussels, Belgium, 2015; ISBN 978-92-823-7854. [CrossRef]

20. Tapscott, D.; Tapscott, A. How blockchain will change organizations. MIT Sloan Manag. Rev. 2017, 58, 10-13.

21. Nofer, M.; Gomber, P.; Hinz, O.; Schiereck, D. Blockchain. Bus. Inf. Syst. Eng. 2017, 59, 183-187. [CrossRef]

22. Xia, Q.; Sifah, E.B.; Smahi, A.; Amofa, S.; Zhang, X. BBDS: Blockchain-based data sharing for electronic medical records in cloud environments. Information 2017, 8, 44. [CrossRef]

23. Haggins, S. United Nations Lab Testing Blockchain for Remittances. 2017. Available online: https://www. coindesk.com/united-nations-blockchain-remittances (accessed on 1 October 2017).

24. Azar, E.; Raouf, M.A. (Eds.) Sustainability in the Gulf-Challenges and Opportunities; Routledge Explorations in Environmental Studies series; Taylor \& Francis Ltd.: Oxfordshire, UK, 2017.

25. Babcock, L.H. What Do the UN's New Sustainable Development Goals Mean for the Blockchain? Digital Currency Council: New York, NY, USA, 2015. Available online: https:/ / www.digitalcurrencycouncil.com/ professional/what-do-the-uns-new-sustainable-development-goals-mean-for-the-blockchain (accessed on 1 October 2017). 
26. Zambrano, R.; Seward, R.K.; Sayo, P. Unpacking the Disruptive Potential of Blockchain Technology or Human Development; Report of International Development Research Centre. International Development Research Centre: Ottawa, ON, Canada, 2017. Available online: http://hdl.handle.net/10625/56662 (accessed on 17 October 2017).

27. Green, J.; Newman, P. Citizen utilities: The emerging power paradigm. Energy Policy 2017, 105, $283-293$. [CrossRef]

28. Extance, A. The future of cryptocurrencies Bitcoin and beyond. Nature 2015, 526, 21-23. [CrossRef] [PubMed]

29. Rutkin, A. Blockchain-based micro grid gives power to consumers in New York. New Sci. 2016. Available online: https://www.newscientist.com/article/2079334-blockchain-based-microgrid-givespower-to-consumers-in-new-york/ (accessed on 5 May 2017).

30. The Declaration of Bitcoin Independence. Available online: https://freebtc.website/blog/declarationBitcoin-independence/ (accessed on 29 May 2017).

31. Golumbia, D. Bitcoin as Politics: Distributed Right-Wing Extremism. In MoneyLab Reader: An Intervention in Digital Economy Amsterdam; Lovink, G., Tkacz, N., De Vries, P., Eds.; Institute of Network Cultures: Amsterdam, The Netherlands, 2015; pp. 117-131.

32. Battanta, L. Chi Governa Il Bitcoin? Tredici Domande Per Capire Quale Vincerà Fra Le Visioni A Confronto. Il Sole 24ore. 5 April 2017. Available online: http:/ / www.ilsole24ore.com/art/finanza-e-mercati/2017-0405/chi-governa-Bitcoin-tredici-domande-capire-quale-vincera-le-visioni-confronto-131100.shtml?uuid= AEqPCqz (accessed on 6 June 2017).

33. Ver, R. How Bitcoin Can Stop War. 2014. Available online: http://original.antiwar.com/roger_ver/2014/07/ 21/how-Bitcoin-can-stop-war/ (accessed on 15 April 2017).

34. Reynolds, P.; Reynolds, P.; Irwin, A.S. Tracking digital footprints: anonymity within the Bitcoin system. J. Money Laund. Control 2017, 20, 172-189. [CrossRef]

35. Vranken, H. Sustainability of bitcoin and blockchains. Curr. Opin. Environ. Sustain. 2017, 28, 1-9. [CrossRef]

36. Kewell, B.; Adams, R.; Parry, G. Blockchain for good? Special Issue: The Future of Money and Further Applications of the Blockchain. Strateg. Chang. Brief. Entrep. Financ. 2017, 26, 429-437. [CrossRef]

37. Kapoor, R. Regulating the Bitcoin Ecosystem. Master's Thesis, Faculty of Technology Policy Management, Delft University of Technology, Delft, The Netherlands, February 2016.

38. Yan, J.S.J.; Zhang, K.Z.K. Blockchain-based sharing services: What blockchain technology can contribute to smart cities. Financ. Innov. 2016, 2, 26. [CrossRef]

39. Faber, N.R.; Hadders, H. Towards a blockchain enabled social contract for sustainability, Creating a fair and just operating system for humanity. In Proceedings of the First International Conference on New Business Models, Toulouse, France, 16-17 June 2016.

40. Ali, M.; Nelson, J.; Shea, R.; Freedman, M.J. Blockstack: A Global Naming and Storage System Secured by Blockchains. In Proceedings of the 2016 USENIX Annual Technical Conference (USENIX ATC '16), Denver, CO, USA, 22-24 June 2016.

41. Republic of Estonia E-Residency. Available online: https://e-resident.gov.ee/ (accessed on 13 October 2017).

(c) 2017 by the authors. Licensee MDPI, Basel, Switzerland. This article is an open access article distributed under the terms and conditions of the Creative Commons Attribution (CC BY) license (http:/ / creativecommons.org/licenses/by/4.0/). 